\title{
Auction-Based Optimal Power Allocation in Multiuser Cooperative Networks
}

\author{
Yuan Liu*, Meixia Tao*, and Jianwei Huang ${ }^{\dagger}$ \\ *Department of Electronic Engineering, Shanghai Jiao Tong University, Shanghai, P. R. China \\ $\dagger$ Department of Information Engineering, The Chinese University of Hong Kong, Hong Kong \\ Email: \{yuanliu,mxtao\}@sjtu.edu.cn, jwhuang@ie.cuhk.edu.hk.
}

\begin{abstract}
This paper considers a wireless network where users cooperate with each other to improve the network performance. Our goal is to design an optimal power allocation algorithm for user cooperation in a distributed fashion. We formulate the power allocation problem as a multi-auctioneer multi-bidder power auction. Specifically, each user acts as both an auctioneer (seller) and a bidder (buyer). Each auctioneer independently determines its trading price and allocates power to bidders, and each bidder chooses the demand from each auctioneer. We show that there exist bidding and pricing strategies that maximize the social welfare (total network throughput). Then we propose a distributed mechanism that can achieve the globally optimal equilibrium. Simulation results verify our proposed approach.
\end{abstract}

\section{INTRODUCTION}

By exploiting the inherent broadcast nature of wireless radio waves, wireless users can cooperate and improve the network throughput and energy efficiency in wireless networks. Although the performance of small cooperative communications has been extensively studied from an information theoretic perspective, there still exist many open problems of realizing its full potential in practical large scale networks.

In this paper, we will design a distributed resource allocation framework for cooperative communications by addressing several key challenges. First of all, forwarding other users' packets consumes valuable resources (e.g., battery energy, transmission slots or bandwidth) and may limit or degrade a user's own performance. Therefore, we need to design a mechanism that makes the autonomous users to cooperate in distributed networks. Second, whether and how to perform cooperative transmission can be a complicated decision depending on channel conditions between nodes. For instance, when the channel condition of the direct link is superior than that of the cooperative link, direct transmission leads to a better throughput. Third, when cooperative communication is desirable, there can be more than one node that is suitable to serve as the relay. Thus, we need to decide how to select the transmission mode (direct or cooperative transmission) and the associated relay nodes for each source transmission. Fourth, if

This work is supported by the Joint Research Fund for Overseas Chinese, Hong Kong and Macao Young Scholars (61028001), the Inter-Government Technology Collaborative Research Project of Shanghai (10220712000), and the Innovation Program of Shanghai Municipal Education Commission (11ZZ19). The work of J. Huang is supported by the General Research Fund (412511) established under the University Grant Committee of the Hong Kong Special Administrative Region, China. a user decides to help others relaying the messages, it needs to balance the resource (such as power, bandwidth, and time slots) reserved for itself and the resource provided for others. Therefore, we need to design a mechanism so that each node captures the optimal tradeoff of resource allocation.

Previous work on resource allocation in cooperative networks fall into two categories: centralized (e.g., [1]-[3]) and distributed (e.g., [4]-[13]). The centralized schemes require global channel state information (CSI) for centralized processing, thus are often not scalable due to the large signalling overhead. Thus, distributed schemes based on local information only are more favorable in practical systems. There have been several results on distributed resource allocation in cooperative networks. For instance, authors in [4] proposed a distributed relay selection scheme based on limited instantaneous signal-to-noise ratio (SNR). In [5], authors investigated the distributed power control for a single source-destination pair with multiple relays to maximize the multihop diversity. A distributed power allocation algorithm using Stackelberg game was presented in [12], where the source is modeled as a buyer and the relay nodes are modeled as sellers. The work in [13] studied an auction-based distributed power allocation scheme for a cooperative network, where multiple users are assisted by a fixed single relay node. Therein, the relay acts as the auctioneer and the users act as the bidders.

Unlike the previous works on relay-assisted networks [10][13] where relays act as sellers and users act as buyers, we propose a new auction-based power allocation framework for multiuser cooperative networks, with each user acting the double roles. By employing distributed multiple-input multipleoutput (MIMO) techniques (e.g., [14], [15]) for cooperative transmission, the proposed power allocation scheme incorporates the transmission mode selection and relay selection. With the double roles, each user can decide whether to cooperate, with whom to cooperate, and how to cooperate in a distributed fashion by evaluating a unified achievable rate expression. Specifically, we design a multi-auctioneer multi-bidder power auction. Each user acts as both an auctioneer and a bidder. Each auctioneer independently announces its trading price and sells power, and each bidder dynamically decides whether to buy, from which auctioneer(s) to buy, and how much to buy. Then, a fully distributed mechanism is designed to achieve the optimum. 


\section{System Model and Problem Formulation}

The network consists of $K$ cooperative users, each of which has its own source node and destination node. Different users have distinct source nodes, but may share the same destination node. Each source node can transmit over multiple sub-bands (or orthogonal channels), one sub-band for its own messages and others for relaying other users' messages. The users can cooperate with each other, thus the source node of a user can be a transmitter and a forwarder simultaneously. Such cooperative transmission can be regarded as a distributed multiple-antenna system. Each node is subject to the halfduplex constraint, so that it cannot transmit and receive at the same time over the same sub-band. However, each node can transmit and receive simultaneously on different sub-bands.

The cooperative transmission includes two phases. In the first phase, the source node of each user transmits the message to its own destination on one sub-band and listens on other sub-bands. In the second phase, some source nodes (who have the proper channel condition and extra transmission power) form a distributed MIMO system on some sub-bands and simultaneously help the transmission of one particular user by using distributed space-time codes. An illustrative example is shown in Fig. 1.

Let $\mathcal{K}=\{1,2, \ldots, K\}$ be the set of users. The $K \times K$ matrix $\boldsymbol{p}$ denotes the transmission power, where $p_{j, i}$ denotes the amount of power that the source node of user $j$ contributes to forwarding user $i$ 's information for $i \neq j$, or the amount of power user $i$ consumes for its own transmission for $i=j$. The sum of the $j$ th row of $\boldsymbol{p}$ represents the total power consumption of user $j$, which is subject to a peak power constraint $\bar{p}_{j}$. This power matrix implicitly accounts for transmission mode and relay node adaptations. For instance, if $\boldsymbol{p}$ is a diagonal matrix, then only direct transmission is selected; full user cooperation is selected if all the elements of $\boldsymbol{p}$ are non-zero.

Let $\boldsymbol{R}$ be a $K \times 1$ vector whose element $R_{i}$ denotes the achievable rate of user $i$ at a given power allocation vector $\left\{p_{j, i}\right\}_{j=1}^{K}$. This achievable rate definition can accommodate different distributed MIMO techniques such as amplify-andforward (AF), decode-and-forward (DF), or compress-andforward (CF) relaying protocols. The detailed expression of rate $R_{i}$ will be given later.

The global network objective is to allocate the power on each source node in order to maximize the network total throughput, also called the social welfare in this paper. The optimization problem can be formulated as follows (P1):

$$
\begin{aligned}
\text { P1: } \max & \sum_{i \in \mathcal{K}} R_{i} \\
\text { s.t. } & \sum_{i \in \mathcal{K}} p_{j, i} \leq \bar{p}_{j}, \forall j \in \mathcal{K} \\
\text { variables } & \boldsymbol{p} \succeq \mathbf{0}
\end{aligned}
$$

If each $R_{i}$ is a concave function of the power vector $\left\{p_{j, i}\right\}_{j=1}^{K}$, then the objective function is concave as any positive linear combination of concave functions is concave.

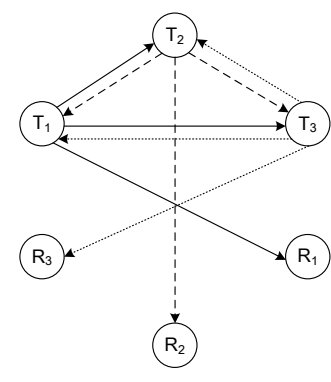

first phase

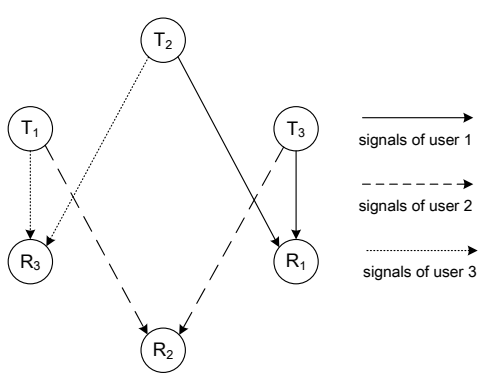

second phase
Fig. 1: An example of the cooperative communication with three users (transmitter-receiver pairs). $T_{i}$ and $R_{i}$ denote the transmitter and receiver of user $i$, respectively.

Moreover, constraint (2) is convex and constraint (3) is affine. Hence the feasible set of this optimization problem is convex. Therefore, $\mathbf{P 1}$ is a convex optimization problem and can be solved easily. The Lagrangian of $\mathbf{P 1}$ is given by:

$$
L(\boldsymbol{p}, \boldsymbol{\lambda})=\sum_{i \in \mathcal{K}} R_{i}-\sum_{j \in \mathcal{K}} \lambda_{j}\left(\sum_{i \in \mathcal{K}} p_{j, i}-\bar{p}_{j}\right),
$$

where $\boldsymbol{\lambda}=\left(\lambda_{1}, \lambda_{2}, \ldots, \lambda_{K}\right) \succeq 0$ are the Lagrange multipliers of the $K$ maximum the power constraints. Applying the Karush-Kuhn-Tucker (KKT) conditions [16], we obtain the following necessary and sufficient conditions for the optimal primal variables $\boldsymbol{p}^{*}$ and dual variables $\boldsymbol{\lambda}^{*}$ :

$$
\begin{aligned}
R_{i}^{\prime}\left(p_{j, i}^{*}\right) & =\lambda_{j}^{*}, \forall p_{j, i}^{*}>0, i, j \in \mathcal{K}, \\
\lambda_{j}^{*}\left(\sum_{i \in \mathcal{K}} p_{j, i}^{*}-\bar{p}_{j}\right) & =0, \forall j \in \mathcal{K}, \\
\sum_{i \in \mathcal{K}} p_{j, i}^{*} & \leq \bar{p}_{j}, \forall j \in \mathcal{K}, \\
\boldsymbol{p}^{*} \succeq \mathbf{0}, \boldsymbol{\lambda}^{*} & \succeq \mathbf{0} .
\end{aligned}
$$

Note that $R_{i}^{\prime}\left(p_{j, i}^{*}\right)=0$ if $p_{j, i}=0$, which can be obtained directly by differentiating the Lagrangian function with respect to $p_{j, i}$.

Next we study the concavity of the achievable rates $\boldsymbol{R}$ with respect to the power allocation matrix $\boldsymbol{p}$. For an illustration purpose, we employ the AF in this paper. We model the wireless fading environment by the large-scale path loss, shadowing, and small-scale Rayleigh fading. The additive white Gaussian noises (AWGN) at all users are assumed to be independent circular symmetric complex Gaussian random variables.

In the first phase of the cooperative communication, each source node $i \in \mathcal{K}$ broadcasts its signal $x_{i}$ to all other source nodes $\forall j \neq i$ and the destination $d$. Thus the received signal at node $j$ and the destination $d$ from $i$ is given by, respectively

$$
y_{i, d}=\sqrt{p_{i, i}} h_{i, d} x_{i}+n_{d},
$$

and

$$
y_{i, j}=\sqrt{p_{i, i}} h_{i, j} x_{i}+n_{j}, \forall j \neq i,
$$


where $n_{d}$ and $n_{j}$ are the AWGN at the destination and node $j$, respectively, $h_{i, d}$ and $h_{i, j}$ are the complex-valued channel gains from node $i$ to the destination and node $j, \forall i, j$, respectively.

In the second phase, let $\mathcal{K}_{i}^{\prime}$ be the set of users who participate in the cooperative transmission of user $i$. Denote $\mathbf{A}_{j}$ be the corresponding space-time coding matrix to be employed by user $j \in \mathcal{K}_{i}^{\prime}$ [14]. Then, each user multiplies its received signals with the space-time code matrix $\mathbf{A}_{j}$ and forwards the signal to the destination using power $p_{j, i}$. The received signal at the destination $d$ is

$$
y_{d}=\sum_{j \in \mathcal{K}_{i}^{\prime}} \sqrt{p_{j, i}} h_{j, d} x_{j, d}+n_{d}^{\prime},
$$

where $x_{j, d}$ is the normalized transmitted signal from node $j$ that contains both user $i$ 's information and amplified noise. By employing the maximal-ratio combining (MRC) for the direct and cooperative links, the achievable rate of user $i$ is

$$
\begin{aligned}
R_{i}=\frac{1}{2} \log _{2}(1 & +p_{i, i}\left|h_{i, d}\right|^{2} \\
& \left.+\sum_{\forall j \in K_{i}^{\prime}} \frac{p_{i, i}\left|h_{i, j}\right|^{2} p_{j, i}\left|h_{j, d}\right|^{2}}{1+p_{i, i}\left|h_{i, j}\right|^{2}+p_{j, i}\left|h_{j, d}\right|^{2}}\right)
\end{aligned}
$$

Note that $R_{i}$ is not jointly concave in $p_{i, i}$ and $p_{j, i}, \forall j \in$ $\mathcal{K}_{i}^{\prime}$. To make the analysis tractable, we adopt the following approximation:

$R_{i} \approx \frac{1}{2} \log _{2}\left(1+p_{i, i}\left|h_{i, d}\right|^{2}+\sum_{j \in \mathcal{K}_{i}^{\prime}} \frac{p_{i, i}\left|h_{i, j}\right|^{2} p_{j, i}\left|h_{j, d}\right|^{2}}{p_{i, i}\left|h_{i, j}\right|^{2}+p_{j, i}\left|h_{j, d}\right|^{2}}\right)$.

Such approximation is commonly used for AF rate expression in the literature for the single-relay case. It can also be proved that $R_{i}$ is strictly concave in the transmission powers by evaluating the Hessian matrix [16]. To be more general, we can replace the relay set $\mathcal{K}_{i}^{\prime}$ by $\mathcal{K} /\{i\}$ in the rate expression (13). This does not change the rate results nor the concavity of $R_{i}$ because for those users $j$ who are not involved in the cooperation we can simply let $p_{j, i}=0$.

In the next section, we propose an auction based distributed algorithm to achieve the optimal solution of $\mathbf{P 1}$.

\section{Auction-Based Power Allocation}

In this section, we first show that there exists a auction equilibrium in the proposed auction game. Then we propose a distributed approach to achieve the optimal equilibrium.

We achieve efficient power allocation problem through a multi-auctioneer multi-bidder power auction. Each user has two roles in the auction: an auctioneer and a bidder. In what follows, we will use "user", "auctioneer", and "bidder" interchangeably. The interaction of the users is illustrated in Fig. 2, in which each bidder dynamically decides whether to buy, from which auctioneer(s) to buy, and how much to buy. In sequence, each auctioneer independently announces its trading price and allocates power to the bidders.

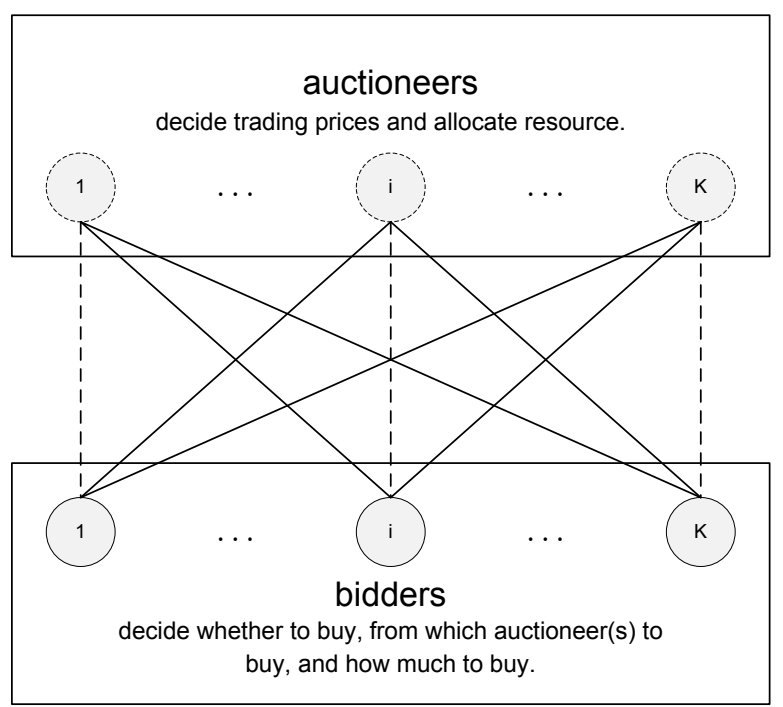

Fig. 2: Illustration of interaction among nodes.

In the proposed approach, each user $i$ submits a bid $b_{j, i}$ to each user $j$. When $i=j$, we assume that user $i$ submits a virtual bid $b_{i, i}$ to itself, since it also acts as an auctioneer (denoted as dotted lines in Fig. 2). By bidding for its own resource, each node $i$ can determine how much power $p_{i, i}$ to consume for itself, besides determining how much power $p_{j, i}$ $(\forall j \neq i)$ it buys from others.

Let $\boldsymbol{\pi}=\left(\pi_{1}, \pi_{2}, \ldots, \pi_{K}\right)$ be the price vector of the auctioneers, and $\boldsymbol{b}$ the bidding matrix whose element $b_{j, i}$ means the willingness bidder $i$ buys from auctioneer $j$. By definition, $\boldsymbol{b}_{i}=\left\{b_{j, i}\right\}_{j=1}^{K}$, the column $i$ of $\boldsymbol{b}$, is user $i$ 's bidding vector. The multi-auctioneer multi-bidder power auction consists of two parts: 1) For a given price vector $\pi$, each bidder $i$, $\forall i$, determines its demand vector $\left\{p_{j, i}\right\}_{j=1}^{K}$, then submits the corresponding bid vector $\left\{b_{j, i}\right\}_{j=1}^{K}$ to auctioneers; 2) For the collected bids $\boldsymbol{b}$, each auctioneer $j, \forall j$, determines its supply vector $\left\{p_{j, i}\right\}_{i=1}^{K}$ and announces its price $\pi_{j}$.

The key challenge is how to design the price vector $\pi$ and bidding matrix $\boldsymbol{b}$ so that the outcome of the proposed power auction is equivalent to the optimal solution of $\mathbf{P 1}$.

We introduce the two-side auction rule: 1) In the side of the bidders, each bidder $i, \forall i$, submits it bid proportionally to the price of auctioneer $j$ and the power it will purchase from auctioneer $j$, i.e., $b_{j, i}=\pi_{j} p_{j, i}, \forall j$. Intuitively, if $p_{j, i}=0$, bidder $i$ does not bid for auctioneer $j$. 2) In the side of the auctioneers, Kelly-mechanism [17] is adopted such that each auctioneer $j, \forall j$, attempts to maximize the surrogate function $\sum_{i \in \mathcal{K}} b_{j, i} \log p_{j, i}$. Note that the surrogate function can be selected arbitrarily if it is differentiable, strictly increasing and concave in $p_{j, i}$.

Proposition 1: There exists an optimal supply vector $\left\{p_{i, j}^{*}\right\}_{j=1}^{K}$ from each auctioneer $i$ and an optimal demand vector $\left\{p_{j, i}^{*}\right\}_{j=1}^{K}$ from each bidder $i, \forall i$, that coincide with P1.

Proof: User $i$ 's achievable rate $R_{i}$ is only related to $\left\{p_{j, i}\right\}_{j=1}^{K}$ and do not have an explicit relationship $\left\{p_{i, j}\right\}_{j=1}^{K}$. Since $R_{i}$ is jointly concave in $\left\{p_{j, i}\right\}_{j=1}^{K}$, bidder $i$ can optimally 
decides its demand $\left\{p_{j, i}^{*}\right\}_{j=1}^{K}$ satisfying (5)-(8) given the optimal dual vector $\lambda^{*}$. From the bipartite graph in Fig. 2, we know that the amount of power bidder $i$ buys from auctioneer $j$ is equivalent to the amount of power auctioneer $j$ sells to bidder $i$. Therefore, optimal demand vectors $\left\{p_{j, i}^{*}\right\}_{j=1}^{K}$ lead to optimal supply vectors $\left\{p_{i, j}^{*}\right\}_{j=1}^{K}$.

Proposition 1 implies that the double roles of each user can be separated without loss of optimality. This is reasonable since we focus on designing a distributed mechanism instead of game theoretical analysis of a distributed system. In other words, the global optimum can be achieved if the users simply follow the proposed mechanism without really computing the local payoff selfishly. We first focus how users maximize their achievable rates as bidders. Then we turn to the analysis of auctioneer role of each user.

It is assumed that the bidders are price-takers, which means they do not choose their bids strategically to impact the auctioneers' prices. This is reasonable when there are many bidders in the market, such that each bidder's impact on the prices is small. Each bidder $i$ maximizes its surplus, which is the difference between the benefit from buying power from auctioneers and its payments. For the given prices $\boldsymbol{\pi}$, bidder $i$ first determines its optimal demand according to the following surplus maximization (Bidder Sub-Problem):

$$
\max _{\left\{p_{j, i}\right\}_{j=1}^{K}} S_{i}=R_{i}-\sum_{j \in \mathcal{K}} \pi_{j} p_{j, i} .
$$

Then bidder $i$ submits its optimal bids to auctioneers according its optimal demand and the given prices $\pi$ :

$$
b_{j, i}^{*}=p_{j, i}^{*} \pi_{j}, \forall j
$$

It is not difficult to prove that the surplus function $S_{i}$ is jointly concave in $\left\{p_{j, i}\right\}_{j=1}^{K}$, where $R_{i}$ (as defined in (13)) is a concave function of $\left\{p_{j, i}\right\}_{j=1}^{K}$. Due to the concavity of $S_{i}$, bidder $i$ can optimally choose the unique power vector $\left\{p_{j, i}^{*}\right\}_{j=1}^{K}$ to maximize its profit. Differentiating $S_{i}$ with respect to $p_{j, i}$, we can obtain the sufficient and necessary first order condition:

$$
\frac{\partial S_{i}}{\partial p_{j, i}^{*}}=R_{i}^{\prime}\left(p_{j, i}^{*}\right)-\pi_{j}=0, \forall p_{j, i}>0, i, j .
$$

Observing (5), the KKT condition of P1, we notice that if auctioneers announce their prices as

$$
\pi_{j}^{*}=\lambda_{j}^{*}=R_{i}^{\prime}\left(p_{j, i}^{*}\right), \quad \forall i, j, \forall p_{j, i}^{*}>0,
$$

then (16) coincides with (5). In this case, the optimal power $\boldsymbol{p}^{*}$ in the bidder sub-problem is consistent with the one in $\mathbf{P 1}$.

From above we can see that the individual optimum in the Bidder Sub-problem coincides with the global optimum if the prices are appropriately selected.

After introducing the Bidder Sub-problem, we now turn to the auctioneers. Solving the optimal power supply of each auctioneer $j$ can be formulated as (Auctioneer Sub-Problem):

$$
\begin{aligned}
\max & \sum_{i \in \mathcal{K}} b_{j, i} \log p_{j, i} \\
\text { s.t. } & \sum_{i \in \mathcal{K}} p_{j, i} \leq \bar{p}_{j} \\
\text { variables } & \boldsymbol{p} \succeq \mathbf{0} .
\end{aligned}
$$

The associated Lagrangian can be written as

$$
L_{j}^{\prime}=\sum_{i \in \mathcal{K}} b_{j, i} \log p_{j, i}-\mu_{j}\left(\sum_{i \in \mathcal{K}} p_{j, i}-\bar{p}_{j}\right),
$$

where $\mu_{j}$ is the Lagrange multiplier of auctioneer $j$. The KKT conditions for the Auctioneer Sub-Problem are given by

$$
\begin{aligned}
p_{j, i}^{*} & =\frac{b_{j, i}}{\mu_{j}^{*}}, \forall i \in \mathcal{K}, \\
\mu_{j}^{*}\left(\sum_{i \in \mathcal{K}} p_{j, i}^{*}-\bar{p}_{j}\right) & =0 \\
\sum_{i \in \mathcal{K}} p_{j, i}^{*} & \leq \bar{p}_{j}, \\
\boldsymbol{p}^{*} \succeq \mathbf{0}, \mu_{j}^{*} & \geq 0 .
\end{aligned}
$$

By comparing the Auctioneer Sub-Problem with P1, one can see that if $\boldsymbol{\mu}=\boldsymbol{\lambda}$ and bidders select their bids as follows:

$$
b_{j, i}^{*}=p_{j, i}^{*} R_{i}^{\prime}\left(p_{j, i}^{*}\right),
$$

then (22)-(25) are equivalent to (5)-(8) and the solutions of the Auctioneer Sub-Problem for all auctioneers coincide with P1.

We now design a mechanism to realize the multi-auctioneer multi-bidder power auction in a distributed fashion, in which we incorporate primal-dual algorithms which have been studied extensively in the literature. The mechanism is executed iteratively. Formally, we present the detailed mechanism in Algorithm 1. Each iteration consists of power allocation, price update, and bid update (Algorithm 2). Note that Algorithm 2 incorporates auctioneer selection and transmission mode selection.

Proposition 2: The proposed multi-auctioneer multi-bidder power auction algorithm globally converges to the unique globally optimal point $\left(\boldsymbol{p}^{*}, \boldsymbol{b}^{*}, \boldsymbol{\lambda}^{*}\right)$.

The proof is based on LaSalle's invariance principle and the details are omitted here due to the space limitation.

\section{Simulation Results}

A four-user cooperative network with a common destination is simulated. The network topology is a two-dimensional plane of size $1 \times 1 \mathrm{~km}^{2}$, where the common destination $d$ is located at $(0,0)$, and the four transmitting nodes are located at $(0.2,0.5)$, $(0.4,0.3),(0.5,0.8)$, and $(0.8,0.6)$. All nodes have the same maximum power constraints $\bar{p}_{i}=10 \mathrm{~dB}, \forall i \in \mathcal{K}$. We set the path loss exponent to be 3.5 and the standard deviation of log-normal shadowing is $5.8 \mathrm{~dB}$.

Figure 3 shows the dynamics of prices and payoffs of the users, respectively, using step size $\epsilon_{i}=10^{-3}, \forall i \in \mathcal{K}$. The 

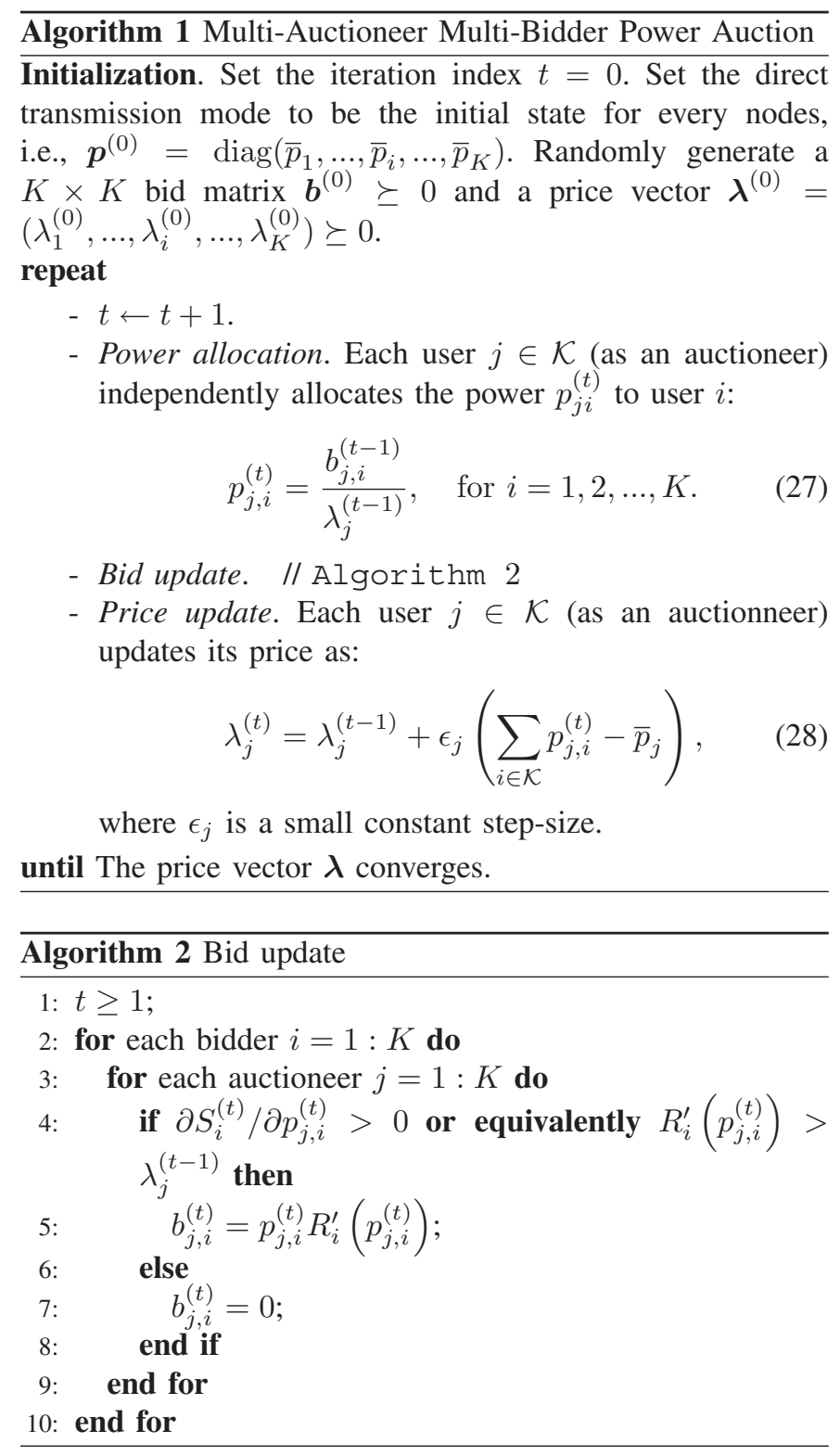

total payoff of user $i$ illustrated in the figure is defined as $R_{i}-\sum_{j \in \mathcal{K}} p_{j, i} \lambda_{j}+\lambda_{i} \sum_{j \in \mathcal{K}} p_{i, j}, \forall i \in \mathcal{K}$, which is the sum profit of the auctioneer and bidder. The figure shows that the prices and payoffs converge to the equilibrium (about 60 iterations in this example). We also see that the closer a node is located to the destination, the higher price it addresses and the higher payoff it achieves. For example, node 1 is the closest to the destination, and thus it has the highest price and highest payoff.

\section{CONCLUSION}

In this paper, we studied the problem of distributed resource allocation in cooperative networks. We solved the problem by mapping it into the multi-auctioneer multi-bidder power auction. By implementing the proposed power auction, each user can achieve the optimal tradeoff of power allocation between its own transmission and forwarding traffic for other
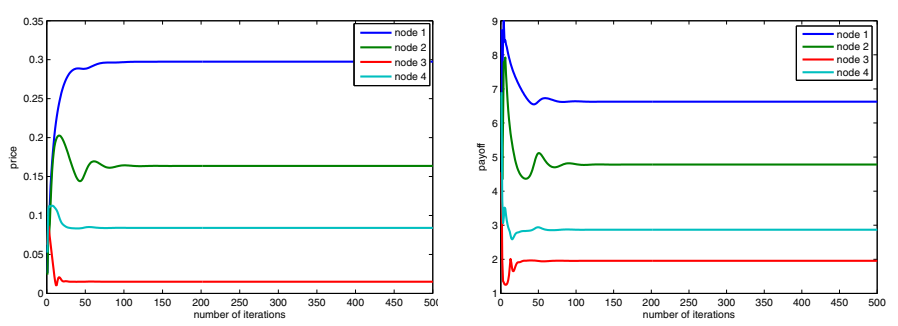

Fig. 3: Convergence speed of prices and payoffs, using $\epsilon_{i}=$ $10^{-3}, \forall i$.

nodes, and users' distributed decisions converge to the global optimal solution. Finally, we propose extensive simulation results that well coincide with the analysis.

\section{REFERENCES}

[1] T. Ng and W. Yu, "Joint optimization of relay strategies and resource allocations in cooperative cellular networks," IEEE J. Sel. Areas Commun., vol. 25, no. 2, pp. 328-339, Feb. 2007.

[2] Y. Liu, M. Tao, B. Li, and H. Shen, "Optimization framework and graph-based approach for relay-assisted bidirectional OFDMA cellular networks," IEEE Trans. Wireless Commun., vol. 9, no. 11, pp. 34903500, Nov. 2010.

[3] W. Dang, M. Tao, H. Mu, and J. Huang, "Subcarrier-pair based resource allocation for cooperative multi-relay OFDM systems," IEEE Trans. Wireless Commun., vol. 9, no. 5, pp. 1640-1649, May 2010.

[4] A. Bletsas, A. Khisti, D. P. Reed, and A. Lippman, "A simple cooperative diversity method based on network path selection," IEEE J. Sel. Areas Commun., vol. 24, no. 3, pp. 659-672, Mar. 2006.

[5] S. Savazzi and U. Spagnolini, "Energy aware power allocation strategies for multihop-cooperative transmission schemes," IEEE J. Sel. Areas Commun., vol. 25, no. 2, pp. 318-327, Feb. 2007.

[6] L. Duan, L. Gao, and J. Huang, "Contract-based cooperative spectrum sharing," in Proc. IEEE DySPAN, May 2011.

[7] Y. Yan, J. Huang, X. Zhong, and J. Wang, "Dynamic spectrum negotiation with asymmetric information," in Proc. GameNets, Apr. 2011.

[8] Y. Yan, J. Huang, X. Z. M. Zhao, and J. Wang, "Sequential bargaining in cooperative spectrum sharing with reputation effect," in Proc. IEEE GLOBECOM, Dec. 2011.

[9] D. Niyato, E. Hossain, and Z. Han, "Dynamic spectrum access in IEEE 802.22-based cognitive wireless networks: A game theoretic model for competitive spectrum bidding and pricing," IEEE Wireless Commun., vol. 16, no. 2, pp. 16-23, Apr. 2009.

[10] S. Ren and M. van der Schaar, "Pricing and distributed power control in wireless relay networks," IEEE Trans. Signal Proc., vol. 59, no. 6, pp. 2913-2925, Jun. 2011.

[11] D. Li, Y. Xu, J. Liu, and J. Zhang, "Relay assignment and cooperation maintenance in wireless networks: A game theoretical approach," IEE/IET Trans. Commun., vol. 4, no. 17, pp. 2133-2144, Nov. 2010.

[12] B. Wang, Z. Han, and K. J. R. Liu, "Distributed relay selection and power control for multiuser cooperative communication networks using stackelberg game," IEEE Trans. Mobile Comput., vol. 8, no. 7, pp. 975990, Jul. 2009.

[13] J. Huang, Z. Han, M. Chiang, and H. V. Poor, "Auction-based resource allocation for cooperative communications," IEEE J. Sel. Areas Commun., vol. 26, no. 7, pp. 1226-1237, Sep. 2008.

[14] Y. Jing and B. Hassibi, "Distributed space-time coding in wireless relay networks," IEEE Trans. Wireless Commun., vol. 5, no. 12, pp. 3524 3536, Dec. 2006

[15] Y. S and R. Schober, "Distributed space-time block coding," IEEE Trans. Commun., vol. 54, no. 7, pp. 1195 -1206, Jul. 2006.

[16] S. Boyd and L. Vandenberghe, Convex Optimization. Cambridge University Press, 2004.

[17] F. P. Kelly, A. K. Maulloo, and D. K. H. Tan, "Rate control for communication networks: shadow prices, proportional fairness and stability," $J$. Oper. Res. Soc., vol. 49, no. 3, pp. 237-252, Mar. 1998. 